\section{Commentary: Risk stratification for patients undergoing surgical ventricular restoration for ischemic cardiomyopathy - it's more than just the preoperative ejection fraction}

\author{
Harold L. Lazar, MD
}

Stratification of risk has become an integral part of determining which patients will benefit the most from an invasive cardiac procedure. In this edition of the Journal, Toso and colleagues ${ }^{1}$ report on the role of biomarkers and echocardiographic-derived parameters of left ventricular (LV) compliance to determine short- and long-term survival and freedom from major adverse cardiovascular events (MACE) following surgical ventricular restoration (SVR). They found that the combined use of the biomarker N-terminal pro-brain natriuretic peptide (NT-proBNP), which reflects the response of cardiomyocytes to increased stretch from high filling pressures, and restrictive filling patterns (RFPs) on echo, a marker of increased LV wall stiffness and reduced compliance resulting in diastolic dysfunction, were the best prognostic indicators for survival and MACE.

The study involved 186 patients undergoing SVR and concomitant coronary artery bypass grafting derived from the Biomarker Plus Study. ${ }^{2}$ The patients were divided into 4 groups depending on the presence of RFP and NTproBNP levels: group I, no RFP and low NT-proBNP; group II, no RFP and high NT-proBNP levels; group III, RFP and low NT-proBNP levels; and group IV, RFP and high NT-pro BNP levels. LV ejection fraction (EF), LV volumes, and LV mass were similar in all 4 groups before SVR. Patients in group IV (RFP and high NT-proBNP levels) had

\footnotetext{
From the Division of Cardiac Surgery, Boston University School of Medicine, Boston, Mass.

Disclosures: The author reported no conflicts of interest.

The Journal policy requires editors and reviewers to disclose conflicts of interest and to decline handling or reviewing manuscripts for which they may have a conflict of interest. The editors and reviewers of this article have no conflicts of interest.

Received for publication Oct 5, 2020; revisions received Oct 5, 2020; accepted for publication Oct 6, 2020; available ahead of print Oct 13, 2020.

Address for reprints: Harold L. Lazar, MD, 80 East Concord St, Boston, MA 02118 (E-mail: harold.1.lazar@gmail.com)

J Thorac Cardiovasc Surg 2022;164:1102-3

$0022-5223 / \$ 36.00$

Copyright (c) 2020 by The American Association for Thoracic Surgery

https://doi.org/10.1016/j.jtcvs.2020.10.021
}

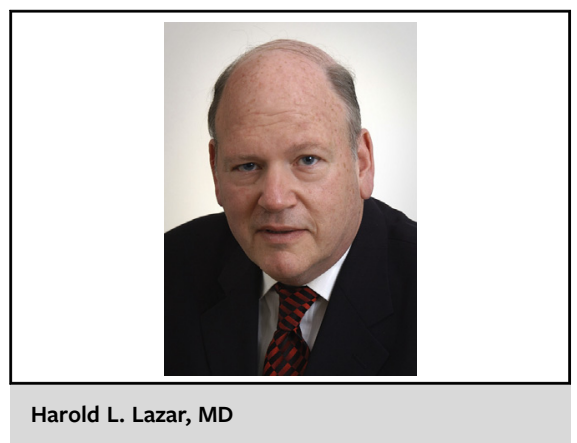

CENTRAL MESSAGE

Biochemical markers and echoderived restriction filling patterns may be more sensitive indicators of postoperative outcomes in patients undergoing surgical ventricular restoration.

significantly increased postoperative mortality and increased mortality at 36 months, a greater incidence of developing any MACE $(71.4 \%)$, and the greatest incidence of readmissions for congestive heart failure $(65 \%)$. The authors concluded that the combination of NT-pro BNP and RFP are sensitive indicators of postoperative morbidity and mortality and can be useful in determining which patients with ischemic cardiomyopathy will derive the most benefit from SVR procedures.

Similar to all post-hoc analyses, this study has several limitations. The sample size is small and NT-proBNP and RFP data are only reported for 6 months following surgery. Therefore, it is unknown whether improvements in these 2 parameters after 6 months might identify other groups of patients who will benefit from SVR who initially presented with RFP and high NT-proBNP levels. The most important limitation is the lack of data regarding the management of coronary artery disease in these patients. The number of patients receiving 2 or more bypass grafts was significantly less in the patients in group IV $(43 \%)$. Furthermore, patients in group IV were less likely to receive a left internal mammary artery-left anterior descending (LAD) graft $(50 \%)$. No data are provided regarding the completeness of revascularization or myocardial viability, especially in the area supplied by the LAD. I strongly suspect that the presence of anterior wall scar and infarct along with the poor quality of the distal LAD was the reason for the decreased incidence of left internal mammary artery to LAD grafting in the patients in group IV. Therefore, the 
inability to revascularize the LAD territory may be another important predictor for increased morbidity and mortality, which is reflected by elevated NT-proBNP levels and the presence of RFP. Finally, the incidence and use of guideline-directed medical therapy (GDMT) is not reported after 6 months. GDMT is an essential component of the treatment of patients with ischemic cardiomyopathy and should be continued indefinitely in patients undergoing SVR. In this study, the overall use of angiotensinconverting enzyme/angiotensin receptor blockers was only $76 \%$. This could have contributed to the greater incidence of readmissions for congestive heart failure in the patients in group IV.

Despite these limitations, the results of this study by Toso and colleagues ${ }^{1}$ show the prognostic importance of biochemical markers and echo-derived parameters of $\mathrm{LV}$ function other than $\mathrm{EF}$, which have been documented in several recent studies. Jacobs and colleagues, ${ }^{3}$ reporting data from cardiac surgery patients in the Northern New England Cardiovascular Study group, demonstrated that elevated levels of NT-proBNP and galectin-3, a key mediator of cardiac fibrosis and remodeling, were associated with a significant increase in 1-year mortality or hospital readmissions. Fukunga and colleagues ${ }^{4}$ found that in patients undergoing coronary artery bypass grafting with an EF $<20 \%$, LV size was a better predictor of mortality and MACE than EF. Patients with similar reductions in EF but with no LV dilatation had significantly better outcomes. In contrast, Michler, ${ }^{5}$ reporting on the 10 -year outcomes from the Surgical Treatment for Ischemic Heart Failure
(STITCH) trial, found that those patients who benefited the most from surgery were patients with more extensive coronary artery disease, reduced EF, and larger endsystolic volumes. The ability to reduce left ventricular end-systolic volume with SVR was more important than the preoperative EF in those patients with larger ventricles in STITCH.

The study by Toso and colleagues ${ }^{1}$ highlights the importance of biochemical markers and RFP in determining risk for cardiac surgery patients. Additional studies should now be undertaken to determine whether optimizing GDMT and achieving complete surgical revascularization with arterial grafts can reverse the negative effects of these biochemical and echo parameters to improve long-term survival and decrease MACE in cardiac surgical patients.

\section{References}

1. Toso S, Castelvecchio S, Menicanti L, Volpe M, Fantini F. Prognostic value of natriuretic peptides and restrictive filling pattern before surgical ventricular restoration. J Thorac Cardiovasc Surg. 2022;164:1092-101.

2. Castelvecchio S, Baryshnikova E, Pina IL, Ambrogi F, Milani V, Tramarin R, et al Longitudinal profile of NT-proBNP levels in ischemic heart failure patients undergoing surgical ventricular reconstruction: the biomarker plus study. Int J Cardiol. 2018;260:24-30.

3. Jacobs JP, Alam SS, Owens L, Parker DM, Rezaee M, Likosly DS, et al. The association between novel biomarkers and 1-year readmissions on mortality after cardiac surgery. Ann Thorac Surg. 2018;106:1122-8.

4. Fukunga N, Ribeiro VP, Lafreniere-Roula M, Manlhiot C, Badiwala MV, Rao V Left ventricular size and outcomes in patients with left ventricular ejection fraction less than 20\%. Ann Thorac Surg. 2020;110:863-70.

5. Michler RE. A decade after the STITCH trial: weaving firm clinical recommendations from lessons learned. J Thorac Cardiovasc Surg. 2019;157:950-7. 